\title{
Application of the apparent diffusion coefficient in magnetic resonance imaging in an assessment of the early response to treatment in Hodgkin's and non-Hodgkin's lymphoma - pilot study
}

\author{
Mateusz Patyk ${ }^{1 C, D, E, F}$, Jacek Kwiatkowski2 ${ }^{2 A, B, D, E}$, Aleksander Pawluś ${ }^{1 A, B, D, E}$, Daniel Hołownia ${ }^{1 C, E}$, Kinga Szymańska ${ }^{10, E}$, \\ Rafał Mazur ${ }^{1 E, F}$, Jurand Silicki ${ }^{1 C, E}$, Roman Badowski ${ }^{1 A, E}$, Dąbrówka Sokołowska-Dąbek ${ }^{1 A, D, E}$, \\ Małgorzata Kuliszkiewicz-Janus ${ }^{2 A, D}$, Urszula Zaleska-Dorobisz ${ }^{1 A, D}$ \\ 'Department of General and Paediatric Radiology, Wroclaw Medical University, Wroclaw, Poland \\ ²Department and Clinic of Haematology, Blood Neoplasms, and Bone Marrow Transplantation, Wroclaw Medical University, Wroclaw, Poland
}

\section{Abstract}

Purpose: Lymphoproliferative neoplasms are the largest and most frequently diagnosed entities in the group of haematological malignancies. The aim of the study was to assess whether apparent diffusion coefficient (ADC) measured on the first day of the second cycle of chemotherapy could be a predictor of prognosis and of the final treatment's outcome.

Material and methods: The study included 27 patients with diagnosed Hodgkin's and non-Hodgkin's lymphoma, who had magnetic resonance (MR) performed with diffusion weighted imaging/apparent diffusion coefficient (DWI/ADC) before and on the first day of the second cycle of chemotherapy. Imaging was performed using a $1.5 \mathrm{~T}$ MR scanner. ADC was measured in lymphoma infiltration in the area of the lowest signal in the ADC map and the highest signal on b 800 images in post-treatment study. After that, the corresponding area was determined in a pre-treatment study and an ADC value was measured.

Results: The difference between ADC values in pre-treatment $\left(\mathrm{ADC}=720 \mathrm{~mm}^{2} / \mathrm{s}\right)$ and post-treatment $\left(\mathrm{ADC}=1059 \mathrm{~mm}^{2} / \mathrm{s}\right)$ studies was statistically significant $(p<0.001)$. Cutoff values for estimating response to treatment were established at the level of ADC $1080 \mathrm{~mm}^{2} / \mathrm{s}$, and ADC to muscle ratio at 0.82 in post-treatment study. Patients with ADC $>752 \mathrm{~mm}^{2} / \mathrm{s}$ before treatment manifested lower probability of progression than patients with $\mathrm{ADC}<752 \mathrm{~mm}^{2} / \mathrm{s}$.

Conclusions: ADC measurement's before treatment and on the first day of the second cycle of chemotherapy can be used as a prognostic marker in lymphoma therapy. ADC values lower than $1080 \mathrm{~mm}^{2} / \mathrm{s}$ and an increase of the ratio after the treatment can be considered as a marker of disease progression.

Key words: lymphoma, Hodgkin's lymphoma, magnetic resonance imaging, diffusion MRI.

\section{Introduction}

Lymphoproliferative neoplasms are the largest and most frequently diagnosed entities in the group of haematological malignancies [1] staging, and response assessment of patients with Hodgkin lymphoma (HL). Over the years an increasing trend of incidence rate of lymphomas has been noticed. Currently it amounts to approximately 20-22 new cases per 100,000 persons per year, according to various registers $[2,3]$.

Correspondence address:

Mateusz Patyk, Department of General and Paediatric Radiology, Wrocław Medical University, 68 M. Curie-Skłodowskiej St., 50-369 Wrocław, Poland,

phone: +4871784 2651, e-mail: mtpatyk@gmail.com

Authors' contribution:

A Study design · B Data collection · C Statistical analysis · D Data interpretation - E Manuscript preparation · F Literature search · G Funds collection 


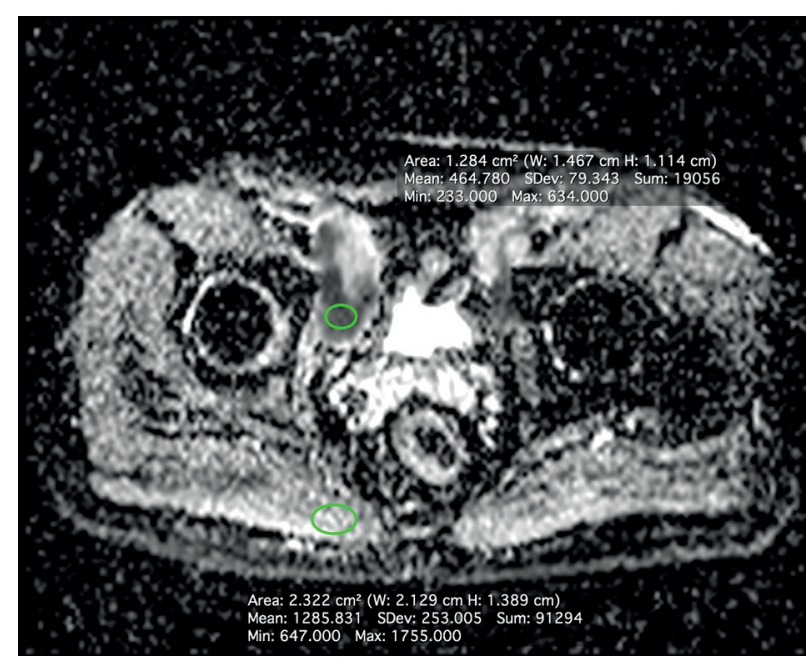

Figure 1. MRI-DWI ADC map in a patient with non-Hodgkin's lymphoma

Table 1. Patients' characteristics $(N=27)$

\begin{tabular}{|l|c|}
\hline Factor & \\
\hline Sex, male/female $(n)$ & $22 / 5$ \\
\hline Age, mean \pm SD (years) & $41.5 \pm 15.6$ \\
\hline Age, median (years) & 41 \\
\hline Hodgkin's lymphoma $(n)$ & 8 \\
\hline Non-Hodgkin's lymphoma $(n)$ & 19 \\
\hline
\end{tabular}

Introducing new diagnostic tools to everyday practice allows more precise evaluation of disease. One of the most important aspects is the evaluation of response to treatment. Accurate assessment of early response is crucial to the diagnosis of lymphoma. This allows patients to be distinguished within the high-risk groups and to modify ineffective treatment in the early stages. This is of utmost importance in the context of individualisation and optimisation of treatment, contributing to positive effects for both the patients and for the entire health care system (economic effect).

Positron emission tomography computed tomography (PET/CT) scanning is currently considered to be the reference method for the assessment of response in the majority of lymphomas, especially in the evaluation of early response to treatment in HL [4] who underwent both $\left[{ }^{18} \mathrm{~F}\right]-F D G-P E T / C T$ and whole-body MRI (including T1- and diffusion-weighted sequences). There are several studies describing the potential role of diffusion weighted imaging (DWI) in the diagnosis and evaluation of response to treatment of lymphomas [5] 31 females, median age -42 years, range $15-86$ years. DWI is a technique in which the image contrast reflects the in vivo changes in the motion of water molecules (Brownian motion) in tissues. A supplemental tool in DWI is the apparent diffusion coefficient (ADC) map, acquired by post-processing of the obtained DWI images [6]. ADC allows for quantitative definition of diffusion parameters (in $\mathrm{mm}^{2} / \mathrm{s}$ ) (Figure 1). The applicability of DWI has been confirmed e.g. in the detection of ischaemic stroke or in the evaluation of breast or prostate gland abnormalities. Numerous recent studies associated with DWI have focused on utilisation of its tools to evaluate response to treatment in oncological patients. Haematological diseases seem to be a very perspective area for the DWI tools, e.g. due to high cellularity of lymphoma infiltration [7].

The purpose of this study was the assessment of the DWI/ADC imaging protocol in the evaluation of the early response to treatment of Hodgkin's and non-Hodgkin's lymphomas. Additionally, we analysed whether the ADC measured on the first day of the second cycle of chemotherapy could be a predictor of prognosis and of the final outcome of the treatment.

\section{Material and methods}

The study included the final group of 27 patients with Hodgkin's and non-Hodgkin's lymphoma diagnosed (Table 1). They underwent MRI of the area in question before the treatment and on the first day of the second cycle of chemotherapy. All examinations were performed using a 1.5 T MR unit with a conventional phased array body coil. The DWI was performed using a standard protocol, namely the single-shot spin-echo-planar imaging (EPI) in the axial plane, with the following parameters: TR 5200$6000 \mathrm{~ms}$, TE - $72 \mathrm{~ms}$, voxel size $2 \times 2 \times 5$, Bw $1448 \mathrm{~Hz} / \mathrm{px}$, b values 50,400 , and $800,30-45$ slices, duration $\sim 6 \mathrm{~min}$.

ADC maps were calculated with a dedicated workstation. ADC values were measured in lymphoma infiltration in the area of the lowest signal in the ADC map images in post-treatment study, paying particular attention to avoid areas that could affect the DWI signal, e.g. haematomas. The corresponding area was determined in the pre-treatment study, and the pre-treatment ADC values were measured afterwards. Only oval-shaped ROIs were used to measure the ADC values, and the size thereof was adjusted to the size of the area with the lowest ADC signal. The ADC values were analysed as an independent value and as a ratio - dorsal muscles were used as the reference organs (Figure 2). A Wilcoxon test was performed to verify the difference between the $\mathrm{ADC}$ values before and after the treatment. The ROC curve was used to determine the cut-off values, and the odds ratio was calculated.

\section{Results}

There was a statistically significant difference between the $\mathrm{ADC}$ values in the pre-treatment $\left(\mathrm{ADC}=720 \mathrm{~mm}^{2} / \mathrm{s}\right)$ and post-treatment $\left(\mathrm{ADC}=1059 \mathrm{~mm}^{2} / \mathrm{s}\right)$ studies (Figure 3 ). The ADC value increased significantly in both groups (Table 2, Figure 4). In the group of patients with diagnosed $\mathrm{HL}$ the ADC increased by $344 \mathrm{~mm}^{2} / \mathrm{s}$ on average, and by $206 \mathrm{~mm}^{2} / \mathrm{s}$ in patients with non-Hodgkin's lymphoma, respectively. The cut-off values used for estimation of the response to the treatment were established at the level 


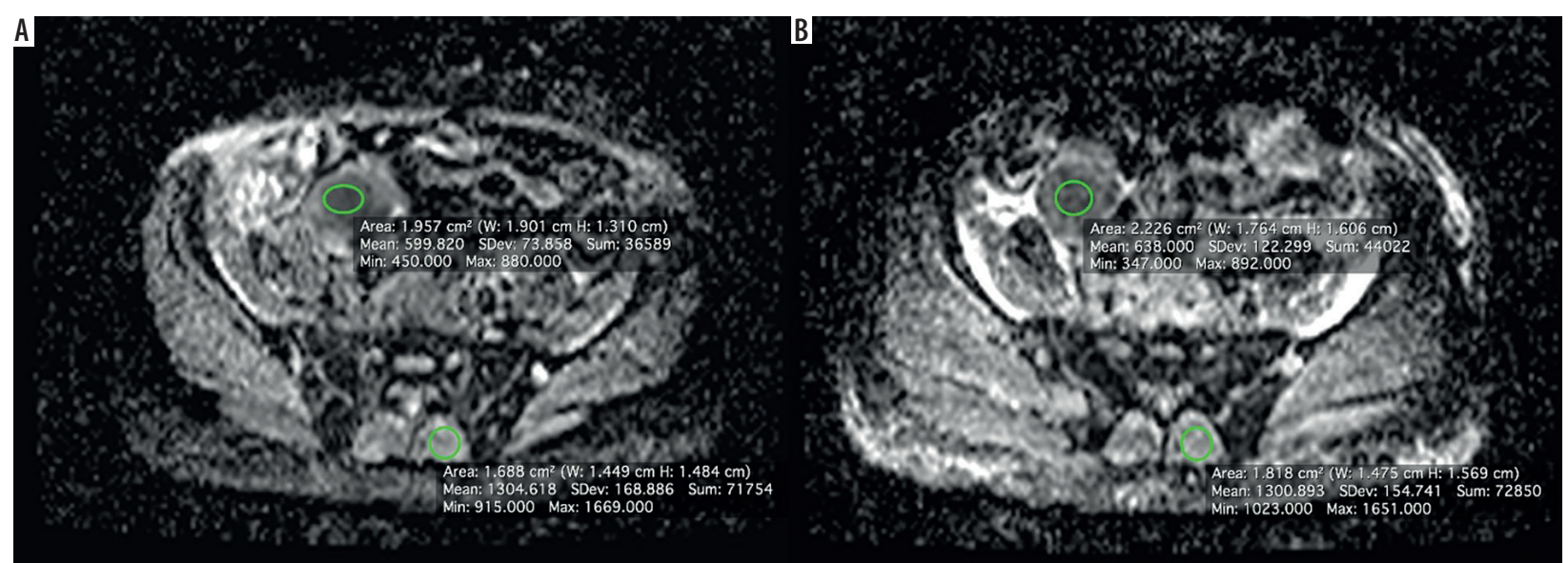

Figure 2. Apparent diffusion coefficient maps. A) Study before treatment, B) study during treatment. 39-year-old male patient with follicular lymphoma. ROls placed in the tumour and in dorsal muscle as a reference. An increase of the ratio of approx. $6 \%$ was calculated. There was a progression of the disease in the follow-up and the patient died

of $\mathrm{ADC} 1080 \mathrm{~mm}^{2} / \mathrm{s}$, and the ADC to muscle ratio 0.82 in the post-treatment study (Figure 3 ). The patients with ADC $>752 \mathrm{~mm}^{2} / \mathrm{s}$ before treatment demonstrated lower probability of progression than the patients with ADC $<752 \mathrm{~mm}^{2} / \mathrm{s}(p=0.046)$. Considering the changes between the studies, an increase of the ADC by $34.5 \%$ and an increase of the ratio of by $32.5 \%$ were determined as the cut-off values. The highest odd ratios were calculated proving that the pre-examination $\mathrm{ADC}$ or the ratio itself would serve best for an assessment of the low response risk.

\section{Discussion}

Advanced imaging techniques play an important role in the diagnosis, evaluation, and staging of lymphomas [1] staging, and response assessment of patients with HL. Despite the fact that the sensitivity and specificity of PET/ $\mathrm{CT}$ with ${ }^{18} \mathrm{~F}-\mathrm{FDG}$ depends on the histological lymphoma subtype, the Lugano classification of malignant lymphomas recommends the use of PET/CT with ${ }^{18} \mathrm{~F}-\mathrm{FDG}$ as the reference imaging technique combined with bone marrow biopsy (BM) $[1,8]$ staging, and response assessment of patients with HL. There are several studies describing the role of whole-body MRI, with the DWI/ADC measurement as a diagnostic tool in the evaluation of patients with lymphoma [9-12] metabolic tumor volume (MTV). The potential role of the measurements of DWI/ADC in patients with non-Hodgkin's lymphoma was well described in 2012 by Chen and Zhong. The authors reported that WB-DWI can be adopted to detect morphological changes of lesions, but moreover it provides important functional information about the growth and decline process of tumour cells [13]. The effectiveness of PET-CT and MRI DWI/ADC in the initial stages of malignant lymphoma was analysed in another study. The authors compared these two methods in a pretherapeutic context and agreement for Ann Arbor staging. They reported high repeat-

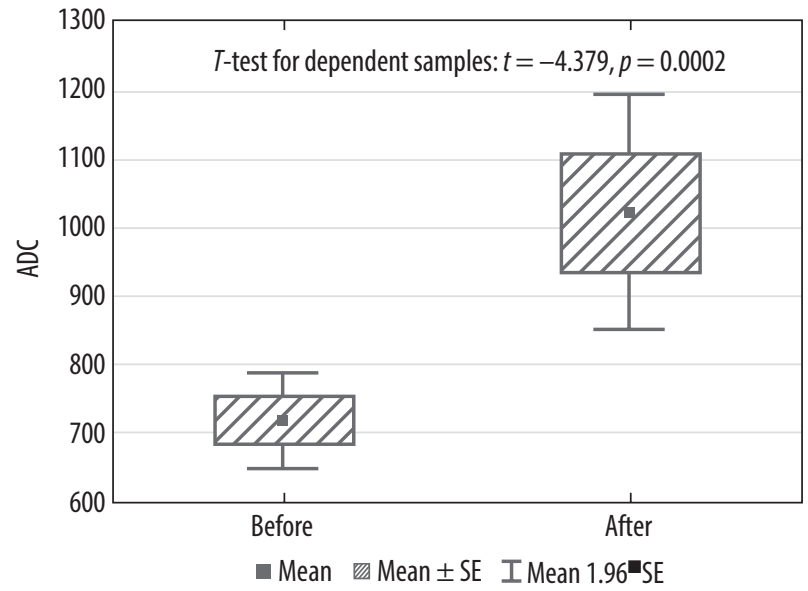

Figure 3. Comparison of the apparent diffusion coefficient (ADC) values before and after the treatment (Student's $t$-test, independent samples)

ability and agreement of MRI DWI/ADC with PET-CT [14]. Different results were stated by investigators from Finland. Wu et al. indicated superiority of the measurements of the standardised uptake value (SUV) of 2-deoxy2 - $\left[{ }^{18} \mathrm{~F}\right]$ fluoro-D-glucose $\left({ }^{18} \mathrm{~F}-\mathrm{FDG}\right)$ against DWI/ADC in patients with diffuse large B-cell lymphoma and follicular lymphoma. There were weak inversed correlations between the $\mathrm{SUV}_{\max }$ and $\mathrm{ADC}_{\min }$ in all cases, but it was not repeated in subgroups [9] MTV. The prognostic feature of the DWI/ADC was the subject of the study performed on the group of 28 patients with primary central nervous system lymphoma. It was investigated which DWI/ADC rank or parameter is a better biomarker of the response to treatment. It was revealed that DWI/ADC $5^{\text {th }}$ percentiles are good predictors for progression-free survival.

The early response to treatment is an important indicator of a patient's condition and prognosis [15]. Appropriately quick assessment enables modification of the treatment protocol and if necessary adjustment to patient's needs. In this study we evaluated weather the ADC measured on the first day of the second cycle of chemotherapy could be a predictor of prognosis and of the final 
Table 2. Apparent diffusion coefficient (ADC) values before and after the treatment of patients with diagnosed Hodgkin's and non-Hodgkin's lymphoma (Student's t-test)

\begin{tabular}{|c|c|c|c|c|}
\hline \multirow[t]{2}{*}{ Parameter } & \multirow[t]{2}{*}{ Total, $N=27$} & \multicolumn{2}{|c|}{ Type of lymphoma } & \multirow[t]{2}{*}{ Avs. C } \\
\hline & & A (non-Hodgkin's), $n=19$ & C (Hodgkin's), $n=8$ & \\
\hline \multicolumn{4}{|c|}{$A D C$ before treatment } & \multirow{4}{*}{$p=0.027$} \\
\hline$M \pm S D$ & $720 \pm 179$ & $768 \pm 176$ & $604 \pm 132$ & \\
\hline $\operatorname{Me}\left(Q_{1} ; Q_{3}\right)$ & $710(572 ; 780)$ & $732(675 ; 840)$ & $571(533 ; 729)$ & \\
\hline Min-Max & $388-1117$ & 480-1117 & $388-780$ & \\
\hline \multicolumn{4}{|c|}{$A D C$ after treatment } & \multirow{4}{*}{$p=0.029$} \\
\hline$M \pm S D$ & $1059 \pm 414$ & $1164 \pm 442$ & $810 \pm 184$ & \\
\hline $\operatorname{Me}\left(Q_{1} ; Q_{3}\right)$ & $980(750 ; 1235)$ & $1115(790 ; 1377)$ & $775(688 ; 963)$ & \\
\hline Min-Max & $549-2098$ & $568-2098$ & $549-1080$ & \\
\hline \multicolumn{4}{|c|}{ Odds ratio before treatment } & \multirow{4}{*}{$p=0.004$} \\
\hline$M \pm S D$ & $0.576 \pm 0.155$ & $0.630 \pm 0.147$ & $0.450 \pm 0.086$ & \\
\hline Me $\left(Q_{1} ; 03\right)$ & $0.53(0.45 ; 0.71)$ & $0.61(0.52 ; 0.77)$ & $0.43(0.40 ; 0.51)$ & \\
\hline Min-Max & $0.32-0.94$ & $0.39-0.94$ & $0.32-0.60$ & \\
\hline \multicolumn{4}{|c|}{ Odds ratio after treatment } & \multirow{4}{*}{$p=0.061$} \\
\hline$M \pm S D$ & $0.791 \pm 0.348$ & $0.872 \pm 0.383$ & $0.599 \pm 0.117$ & \\
\hline $\operatorname{Me}\left(Q_{1} ; Q_{3}\right)$ & $0.65(0.56 ; 1.07)$ & $0.79(0.61 ; 1.23)$ & $0.58(0.53 ; 0.65)$ & \\
\hline Min-Max & $0.11-1.54$ & $0.11-1.54$ & $0.45-0.82$ & \\
\hline
\end{tabular}

$M$ - mean value, SD - standard dimension, Me - median, $Q 1$ - lower quartile, $Q 3$ - upper quartile, Min - minimum value, Max - maximum value, $p$ - $p$-value
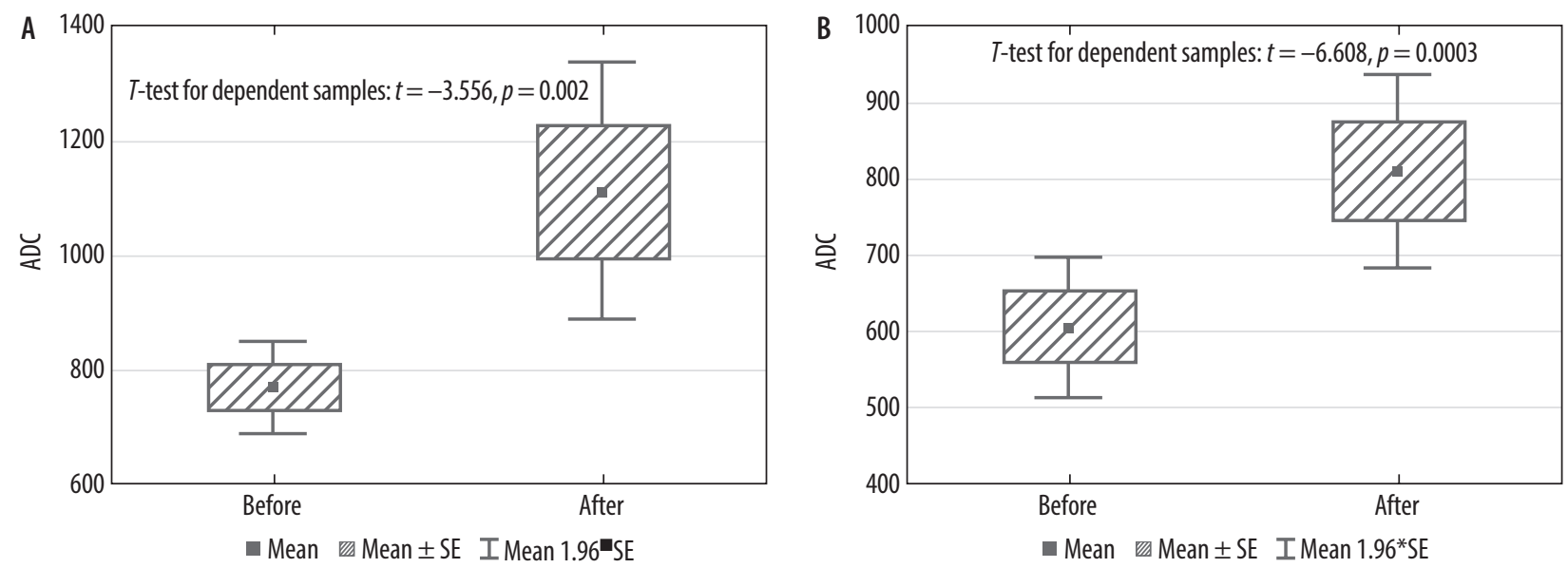

Figure 4. Comparison of the apparent diffusion coefficient (ADC) values before and after the treatment in groups with non-Hodgkin's lymphoma (Group A) and Hodgkin's lymphoma (Group C) (Student's t-test, samples)

treatment's outcome. There was a statistically significant difference between the DWI/ADC values in groups with Hodgkin's and non-Hodgkin's lymphomas. Patients with HL had higher values of DWI/ADC before and after the treatment (respectively, $p=0.027$ and $p=0.029$ ). Similar results were obtained in other studies $[1,16,17]$ i.e., indolent versus aggressive lymphoma, and also to assess the prognostic value of different quantitative parameters of whole-body. Our results indicated that patients with $\mathrm{ADC}>752 \mathrm{~mm}^{2} / \mathrm{s}$ before treatment demonstrated lower probability of progression than the patients with ADC
$<752 \mathrm{~mm}^{2} / \mathrm{s}(p=0.046)$. Mosavi et al. similarly reported a significant relationship between higher mean ADC and longer overall survival $(p=0.006)[16]$. An increase of the ADC by $34.5 \%$ after the second cycle of chemotherapy correlates with a better prognosis. This result has not been confirmed in other cancers. Multivariate analysis in head and neck cancer revealed that lower pre-treatment ADC was associated with a better response to treatment [16].

There were some limitations to this pilot study, such as the small number of patients with diagnosed non-Hodgkin's lymphoma, or lack of histopathological results. 
Moreover, a whole-body MRI was not available at the time of examination and MRI was performed only within the range of interest.

\section{Conclusions}

Measurements of the ADC values before treatment and on the first day of the second cycle of chemotherapy can be used as a prognostic marker in the therapy of lymphomas. The most promising tool for assessing response to treatment seems to be the ratio between the ADC value measured in the area of infiltration and the ADC value of the reference organ (in our case - dorsal muscles). We calculated that an increase of the ratio lower than $32.5 \%$ could serve as a poor prognostic factor and could lead to modification of treatment. Early DWI/ADC measurements enable shortening of the diagnostic process, thus obtaining a quicker assessment of prognosis. An early response to treatment can influence further therapy and can potentially increase the chances for regression of lymphoma. The results seem to be promising, but further studies with larger groups of patients and long-term follow-up are essential to prove the usefulness of the DWI/ADC measurements in the evaluation of lymphoma.

\section{Conflict of interest}

The authors report no conflict of interest.

\section{References}

1. Cheson BD, Fisher RI, Barrington SF, et al. Recommendations for initial evaluation, staging, and response assessment of hodgkin and non-hodgkin lymphoma: The lugano classification. J Clin Oncol 2014; 32: 3059-3067.

2. Campo E, Swerdlow SH, Harris NL, et al. The 2008 WHO classification of lymphoid neoplasms and beyond : evolving concepts and practical applications The 2008 WHO classification of lymphoid neoplasms and beyond : evolving concepts and practical applications. 2014; 117: 5019-5033.

3. Juweid ME, Stroobants S, Hoekstra OS, et al. Use of positron emission tomography for response assessment of lymphoma: Consensus of the imaging subcommittee of international harmonization project in lymphoma. J Clin Oncol 2007; 25: 571-578.

4. Asenbaum U, Nolz R, Karanikas G, et al. Evaluation of [18F]-FDGbased hybrid imaging combinations for assessment of bone marrow involvement in lymphoma at initial staging. PLoS One 2016; 11: $1-12$.

5. Albano D, Patti C, La Grutta L, et al. Comparison between whole-body MRI with diffusion-weighted imaging and PET/CT in staging newly diagnosed FDG-avid lymphomas. Eur J Radiol 2016; 85: 313-318.

6. Azzedine B, Kahina MB, Dimitri P, et al. Whole-body diffusionweighted MRI for staging lymphoma at 3.0T: Comparative study with MR imaging at 1.5T. Clin Imaging 2015; 39: 104-109.

7. Lin C, Itti E, Luciani A, et al. Whole-body diffusion-weighted imaging in lymphoma. Cancer Imaging 2010; 10: 172-178.

8. Johnson SA, Kumar A, Matasar MJ, et al. Imaging for Staging and Response Assessment in Lymphoma. Radiology 2015; 276: 323-338.

9. Wu X, Pertovaara H, Korkola P, et al. Correlations between functional imaging markers derived from PET/CT and diffusion-weight- ed MRI in diffuse large B-cell lymphoma and follicular lymphoma. PLoS One 2014; 9: 1-8.

10. Kwee TC, Takahara T, Ochiai R, et al. Diffusion-weighted wholebody imaging with background body signal suppression (DWIBS): Features and potential applications in oncology. Eur Radiol 2008; 18: 1937-1952.

11. Kwee TC, Basu S, Torigian DA, et al. Evolving importance of diffusion-weighted magnetic resonance imaging in lymphoma. PET Clin 2012; 7: 73-82.

12. Padhani AR, Koh DM, Collins DJ. Whole-Body Diffusion-weighted MR Imaging in Cancer: Current Status and Research Directions. Radiology 2011; 261: 700-718.

13. Chen $\mathrm{Y}$, Zhong J, Wu H, et al. The clinical application of whole-body diffusion-weighted imaging in the early assessment of chemotherapeutic effects in lymphoma: The initial experience. Magn Reson Imaging 2012; 30: 165-170.

14. Stéphane V, Samuel B, Vincent D, et al. Comparison of PET-CT and magnetic resonance diffusion weighted imaging with body suppression (DWIBS) for initial staging of malignant lymphomas. Eur J Radiol 2013; 82: 2011-2017.

15. Frampas E. Lymphomas: Basic points that radiologists should know. Diagn Interv Imaging 2013; 94: 131-144.

16. Mosavi F, Wassberg C, Selling J, et al. Whole-body diffusion-weighted MRI and 18F-FDG PET/CT can discriminate between different lymphoma subtypes. Clin Radiol 2015; 70: 1229-1236.

17. Usuda K, Maeda S, Motono N, et al. Diffusion weighted imaging can distinguish benign from malignant mediastinal tumors and mass lesions: Comparison with positron emission tomography. Asian Pacific J Cancer Prev 2015; 16: 6469-6475. 